ИЗВЕСТИЯ АКАДЕМИИ НАУК ЭСТОНСКОН ССР. ФИЗИКА * МАТЕМАТИКА

PROCEEDINGS OF THE ACADEMY OF SCIENCES OF THE ESTONIAN SSR. PHYSICS * MATHEMATICS

$1985,34,3$

\title{
ОПРЕДЕЛЕНИЕ ОДНОРОДНЫХ СПЕКТРОВ И ФУНКЦИИ НЕОДНОРОДНОГО РАСПРЕДЕЛЕНИЯ МЕТОДОМ ВЫЖИГАНИЯ ПРОВАЛА
}

\author{
(Представил К. К. Ребане)
}

\section{Введение}

K настоящему времени хорошо известно, что дефекты кристалла или неупорядоченное строение стеклообразных веществ обусловливают неоднородное уширение спектров примесных центров. Поэтому для извлечения информации об электронно-колебательном взаимодействии в примесном центре, которая, в принципе, содержится в вибронных спектрах $\left[{ }^{1}\right]$, применяются методы, позволяющие отчасти устранять неоднородное уширение. К таковым относятся селективное лазерное возбуждение флуоресценции $\left[{ }^{2}\right]$ и фотохимическое выжигание провала $\left[{ }^{3,4}\right]$. Появление при этом узких бесфононных линий позволило изучить весьма тонкие аспекты взаимодействия примесной молекулы с матрицей [ $\left.{ }^{5}\right]$.

Однако, как показали модельные расчеты $\left[{ }^{6-8}\right]$, даже этими методами не всегда удается измерить однородный контур спектра непосредственно. Это относится прежде всего к сильно неоднородным системам, в которых ширина распределения частот электронного перехода сравнима с шириной фононного крыла (ФК) или превышает ее. Поэтому было предложено несколько специальных способов определения однородных спектральных контуров и функции неоднородного распределения (ФНР) [ $\left.{ }^{9-11}\right]$.

В настоящей работе разработана методика, особенно подходящая для определения однородных характеристик спектров при сильном неоднородном уширении, которое свойственно большим органическим молекулам в стеклообразных матрицах. При этом исходят из распространенной модели (введенной в $\left[{ }^{6}\right]$ и хорошо оправдавшей себя в дальнейшем $\left.\left[{ }^{12,13}\right]\right)$, предполагая, что неоднородно распределены только частоты электронных переходов. Затем, имея в виду, что различные реализации взаимодействия примеси с твердым окружением в общем могут обусловливать вариации всех измеряемых величин, модель обобщают. Особое внимание уделяют и тому, чтобы методика была экспериментально несложной и нозволила по нескольким измерениям определить сопряженные однородные спектры люминесценции и поглощения, а также ФНР.

\section{Модель}

Следуя $\left[{ }^{6}\right]$, считаем, что неоднородный состав примесных центров выражается только в разных сдвигах их однородных спектров люминесценции $\varphi\left(v_{f}\right)$ и поглощения $x\left(v_{e}\right)$, причем распределение по сдвигам опре- 
деляется ФНР $\varrho(\omega)$. Тогда интенсивность люминесценции, возбуждаемой на частоте $v_{e}$, дается формулой

$$
I\left(v_{f}, v_{e}\right)=C \int_{-\infty}^{\infty} \varphi\left(v_{f}-\omega\right) \chi\left(v_{e}-\omega\right) \varrho(\omega) d \omega .
$$

Далее предположим, что у примесных центров возможны необратимые однофотонные фотохимические превращения. При возбуждении такой системы монохроматическим светом на частоте $v_{b}$ ее ФНР будет изменяться во времени по закону

$$
\varrho(\omega, t)=\varrho_{0}(\omega) \exp \left[-I_{b} x\left(v_{b}-\omega\right) \eta t\right],
$$

где $I_{b}$ - плотность возбуждения, $\eta-$ квантовый выход фотохимического превращения.

Низкотемпературные однородные спектры представим в виде сумм узких чисто электронных линий (ЧЭЛ) и более широких ФК (индексы $l$ и $ш$ соответственно)

$$
\begin{gathered}
\varphi\left(v_{f}\right)=\alpha_{f} \varphi_{l}\left(v_{f}\right)+\left(1-\alpha_{f}\right) \varphi_{w}\left(v_{f}\right), \\
\chi\left(v_{e}\right)=\sigma\left[\alpha_{e} \chi_{e}\left(v_{c}\right)+\left(1-\alpha_{e}\right) \chi_{w}\left(v_{e}\right)\right],
\end{gathered}
$$

где $\sigma-$ интегральное сечение поглощения, $\alpha_{e}, \alpha_{f}-$ факторы ДебаяВаллера спектров поглощения и люминесценции соответственно. Все функции в правых частях равенств (3) и (4), описывающие форму однородных спектров, считаются нормированными на единицу.

\section{Определение однородных спектров}

В пренебрежении перекрытием ЧЭЛ и ФК (что оправдано при достаточно низкой температуре $\left[{ }^{1}\right]$ ) временная зависимость наблюдаемого спектра люминесценции на основе формул (1)-(4) следующая:

$$
\begin{aligned}
& I_{f}\left(v_{f}, t\right)=C_{\sigma}\left\{\alpha_{e} \int_{-\infty}^{\infty} d_{\omega} \varphi x_{l} \varrho_{0} \exp \left[-I_{b} \sigma \alpha_{e} x_{l} \eta t\right]+\right. \\
& \left.+\left(1-\alpha_{e}\right) \int_{-\infty}^{\infty} d_{\omega} \varphi x_{w} \varrho_{0} \exp \left[-I_{b} \sigma\left(1-\alpha_{e}\right) x_{w} \eta t\right]\right\},
\end{aligned}
$$

где аргументы функций $\varphi, x$ и $\varrho$ опущены.

Два компонента спектра в (5) убывают с характеристическими скоростями в отношении $r_{l} / r_{w} \approx\left(\Gamma_{w} / \Gamma_{l}\right) \alpha_{e}\left(1-\alpha_{e}\right)^{-1}$ (см. также [ $\left.\left.{ }^{8}\right]\right)$, где $\Gamma_{l}$ и $\Gamma_{w}$ - ширины линии и крыла соответственно. При не очень больших стоксовых потерях $\left(\alpha_{e} \geqslant 0,1\right)$ типичное отношение $r_{l} / r_{w} \sim 10^{4}$. Это позволяет пренебречь выжиганием через ФК, т. е. считать второй интеграл в (5) независящим от времени

В первой свертке при малых временах выжигания, когда экспоненциальный множитель в подынтегральном выражении близок к единице, узкую ЧЭЛ $x_{l}\left(v_{e}-\omega\right)$ можно принять за $\delta$-функцию относительно $\varrho_{0}(\omega)$ и широкой части спектра люминесценции $\varphi_{w}\left(v_{f}-\omega\right)$. ЧЭЛ спектра люминесценции $\varphi_{l}\left(v_{f}-\omega\right)$ преобразуется также в относительно широкую линию, если ширина $\Gamma_{A}$ аппаратной функции регистрации $A_{f}(v)$ намного больше $\Gamma_{l}$. При этом в выражениях (1) и (5) однородный спектр $\varphi\left(v_{f}-\omega\right)$ заменяется функцией

$$
\Phi\left(v_{f}-\omega\right)=\int_{-\infty}^{\infty} A_{f}\left(v_{f}-v\right) \varphi(v-\omega) d v .
$$

В итоге из (5) получим: 


$$
I_{f}\left(v_{f}, t\right)=C \sigma\left[\left(1-\alpha_{e}\right) K\left(v_{f}\right)+\alpha_{e} \Phi\left(v_{f}-v_{e}\right) \varrho_{0}\left(v_{e}\right) R\left(r_{l} t\right)\right],
$$

где функция

$$
K\left(v_{f}\right)=\int_{-\infty}^{\infty} \Phi\left(v_{f}-\omega\right) \chi_{w}\left(v_{e}-\omega\right) Q_{0}(\omega) d \omega
$$

описывает форму т. н. псевдокрыла, а

$$
R\left(r_{l} t\right)=\int_{-\infty}^{\infty} x_{l}(v) \exp \left[-I_{b} \sigma \alpha_{e} \varkappa_{l}(v) \eta t\right] d v
$$

является монотонно убывающей функцией времени,

Однородный спектр излучения получают в результате следующей процедуры. Запись спектров производится дважды при столь слабом монохроматическом возбуждении, что выжиганием в ходе измерения можно пренебречь, а между двумя измерениями осуществляется выжигание провала на той же частоте возбуждения $v_{b}=v_{e}$. После этого вычисляется разность измеренных спектров, которая на основе (7)-(9) равна

$$
I_{f}\left(v_{f}, 0\right)-I_{f}\left(v_{f}, t\right)=C_{\sigma \alpha_{e}} \Phi\left(v_{f}-v_{e}\right) \varrho_{0}\left(v_{e}\right)\left[1-R\left(r_{l} t\right)\right]
$$

Это выражение пропорционально измеренному с аппаратной функцией $A_{f}(v)$ однородному спектру люминесценции $\Phi\left(v_{f}-v_{e}\right)$, частота ЧӘЛ которого совпадает с частотой возбуждения $v_{e}$.

Определение однородного спектра поглощения происходит аналогично, с использованием разности двух спектров возбуждения люминесценции, причем теперь частота выжигания совпадает с частотой регистрации. Заметим, что аппаратная функция возбуждения $A_{e}(v)$ может быть учтена аналогично (6) заменой $x\left(v_{e}\right) \rightarrow \int_{-\infty}^{+\infty} x(v) A_{e}\left(v_{e}-v\right) d v$.

\section{Обсуждение}

Обсудим сначала правомерность сделанных выше упрощений. В принципе самое «узкое» место в используемой модели - описание неодно-

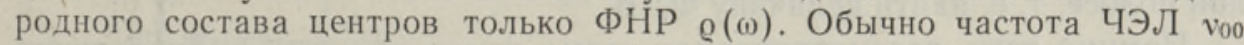
определяется индивидуальными свойствами примеси и сдвиги ее $\omega$, обусловленные локальными полями матрицы, малы (отношение $\omega / v_{0}$ по порядка $1 \%$ или меньше). В колебательной структуре спектра, в которой взаимодействие примеси с матрицей проявляется прямым образом, можно ожидать более значительных вариаций. При учете последних разностный спектр, измеренный согласно вышеописанной процедуре, оказывается средним по однородным спектрам примесных центров с одинаковой $0-0$ частотой. * Соответственно использование термина «однородный» к разностным спектрам является условным в той мере, в какой различаются истинные однородные спектры выделяемых центров. Существование неоднородных вариаций (кроме частот электронного перехода) обнаружено у частот локальных колебаний $\left[{ }^{14}\right] ;$ и у ФДВ $\left[{ }^{15}\right]$. Несмотря на указанное, неизбежное при изучении ансамбля примесных центров, усреднение, достигаемое данной методикой полная селекция по частоте ЧЭЛ качественно повышает информативность измеряемых спектров.

Основная причина использования нами упрощенной модели - избежание громоздких выражений, а явная необходимость в ее обобщении

* Весами при усреднении являются вероятности возбуждения (спектр люминесценции) или регистрации излучения (спектр возбуждения). 
возникает в конкретных расчстах, где начальными данными являются измеренные однородные спектры (см. (15)). Отличительной чертой использованной модели служит возможность выведения относительно простых критериев, определяющих пределы применимости и точность методики. Первая из них связана с тем, что в действительности чисто электронный провал не описывается $\delta$-функцией, т. е. частоты ЧЭЛ выделяемых центров не строго одинаковы. При этом дисперсия частот увеличивается со временем: проявляется эффект уширения провала из-за т. н. фотохимического насыщения $\left[{ }^{16}\right]$. При упрощении первого интеграла в (5) мы считали эту неопределенность меньше требуемого спектрального разрешения. Предлагая лоренцеву форму ЧЭЛ, это условие можно записать в виде неравенства

$$
I_{b} t / D \ll\left(\Gamma_{A} / 2 \Gamma_{l}\right)^{2},
$$

где $D=\pi \Gamma_{l} / 2 \sigma \alpha_{e} \eta$. Пренебрежение процессами выжигания нерезонансных центров через ФК при выводе формулы (7) также налагает верхний предел на плотность дозы облучения $I_{b} t$. Учитывая линейный член в разложении второго интеграла (5) по $r_{w} t$ получим оценку:

$$
\frac{I_{b} t}{D\left[1-R\left(I_{b} t / D\right)\right]} \ll\left(\frac{\alpha_{e}}{1-\alpha_{e}}\right)^{2} \cdot \frac{\Gamma_{w}}{\Gamma_{l}} .
$$

Из (12), в частности, следует, что последнее приближение оправдано у систем не со слишком малыми стоксовыми потерями (ориентировочно при $\left.\alpha_{e} \geqslant 0,1\right)$.

Из-за большого соотношения ширин ЧЭЛ и ФК разделение их является удобной не только при модельном расчете, но и в эксперименте. Предложенная в данной работе методика особенно подходит именно для исследования колебательной структуры спектров, когда не требуется сверхвысокого спектрального разрешения. Кроме того, в случае относительно плохого разрешения $\left(\Gamma_{l} \ll \Gamma_{A}\right)$, когда, однако, линия и крыло в спектре разделяются $\left(\Gamma_{A} \ll \Gamma_{w}\right)$, согласно (10) можно определить интегральную интенсивность ЧЭЛ в отношении ко всей однородной полосе, т. е. найти величину ФДВ.

Помимо того, рассматриваемая схема измерения весьма аналогична определению однородных контуров ЧЭЛ по форме провала - в обоих случаях выделяется однородный по частоте чисто электронного перехода подансамбль примесных центров. Это позволяет утверждать, что и в данном случае промежуточное выжигание может быть обусловлено другими механизмами, например, изменением окружения примеси в стеклах $\left[{ }^{17}\right]$ или накоплением центров в долгоживущее (триплетное) состояние $\left[{ }^{18}\right]$. В $\left[{ }^{19}\right]$ демонстрируется измерение однородных спектров флуоресценции перилена в матрицах Шпольского с использованием фотофизического провала насыщения. Пример измерения разностного спектра медленным промежуточным выжиганием приведен в $\left[{ }^{20}\right]$, где исследованы спектры флуоресценции перилена в пленке. В последнем случае, однако, неясѐн вклад нерезонансных центров.

Наконец, обратим внимание на еще одно упрощение в изложенном выше расчете. Именно в нем не учитывается спектр фотопродукта, который может перекрываться с подлежащим определению однородным спектром. Отметим, что при сильном неоднородном уширении, когда обычно частоты ЧЭЛ примесных центров до и после «прыжка» в другую конфигурацию нескоррелированы, пренебрежение этим эффектом вполне допустимо: погрешность получается порядка той, которая возникла при пренебрежении выжиганием через ФК. 


\section{Определение ФНР}

В рассмотренной выше модели неоднородный спектр люминесценции (при «белом» возбуждении) выражается в виде

$$
J\left(v_{j}\right)=C^{\prime} \int_{-\infty}^{\infty} \Phi\left(v_{j}-\omega\right) \varrho(\omega) d \omega
$$

Если известен однородный спектр люминесценции, из (13) (измерив предварительно $\left.J\left(v_{f}\right)\right)$ можно вычислить ФНР. ** Хотя формально это классическая задача исключения влияния аппаратной функции, опишем алгоритм решения (13), с помощью которого совместно с измерением однородных спектров удобно найти и ФНР.

Пусть определены дискретные массивы

$$
\begin{array}{rlrl}
J_{i} & \equiv J\left(v_{i}\right), \quad v_{i}=v_{0}+(i-1) \Delta, \quad i=1, \ldots ; M ; \\
\langle\Phi\rangle_{k}^{j} \equiv\langle\Phi\rangle^{j}\left(v_{k}\right), \quad v_{k}=-(k-1) \Delta, \quad k=1, \ldots, N .
\end{array}
$$

Верхний индекс в (15) указывает на то, что нормированный на единицу спектр $\left\{\langle\Phi\rangle_{k}{ }^{j} ; k=1, \ldots, N\right\}$ принадлежит примесным центрам с частотой $0-0$ перехода $v_{j}=v_{0}+(j-1) \Delta(j=1, \ldots, M)$, а знак усреднения - на то, что это их средний однородный спектр. Теперь, согласно вышеприведенной методике, $\langle\Phi\rangle_{k}{ }^{j}$ определяется из измеренных разностных спектров. При этом связь с неоднородным спектром остается прежней, т. е. усреднение $\langle Ф\rangle_{k}{ }^{j}$ по ФНР дает $J_{i}$

$$
J_{i}=\sum_{j=i}^{M} n_{j}\langle\Phi\rangle_{j-i+1}^{j}, \quad i=1, \ldots, M
$$

Здесь $n_{j}$ - относительное число примесных центров с положениями ЧЭЛ в окрестности $\Delta$ частоты $v_{j}$. Система (16) представляет собой дискретный аналог уравнения (13) с учетом усложнения модели (Ф $\left(v_{k}\right)$ заменено на $\left.\langle\Phi\rangle^{j}\left(v_{k}\right)\right)$, причем величины $n_{j}$ определяют квазинепрерывную ФНР. Поскольку система (16) является треугольной, можно сразу выписать ее решение в рекуррентном виде:

$$
\left\{\begin{array}{l}
n_{M}=\frac{J_{M}}{\langle\Phi\rangle_{1}^{M}} \\
n_{j}=\frac{1}{\langle\Phi\rangle_{1}^{j}}\left(J_{j}-\sum_{i=j+1}^{M} n_{i}\langle\Phi\rangle_{i-j+1}^{i}\right), \quad j=M-1, \ldots, 1 .
\end{array}\right.
$$

Благодаря сделанному обобщению модели методическую ошибку результата можно считать минимальной. В отношении устойчивости решения самым простым критерием, по-видимому, выступает непосредственный расчет ФНР по экспериментальным или модельным кривым с шумом. При этом, рассматривая спектры (14) и (15) как случайные величины, на основе (16) и аналогичной связи между средними значениями можно получить некоторую предварительную информацию. В частности, случайные разбросы в вычисляемой ФНР $\Delta n_{j}$ окажутся линейно зависимыми от шума в спектрах (относительно маленькие случайные части с нулевыми первыми моментами), так что в случае гауссового шума и $\Delta n_{j}$ распределены по нормальному закону. Относительно высокая точность расчетов по описанной схеме, как отмечено и в $\left[{ }^{11}\right]$, где рассмат-

** Аналогичным образом ФНР можно найти с помощью однородного и неоднородного спектров поглощения. 
ривается формально аналогичный алгоритм деконволюции селективно возбуждаемых спектров, обеспечивается, во-первых, минимальным объемом вычислений в случае треугольной системы уравнений, и, во-вторых, тем, что диагональные элементы матрицы системы (16) на много больше всех других (они равны интенсивностям ЧЭЛ). Здесь надо отметить, что из-за весьма большого отношения неоднородной и однородной ширин интенсивность ЧЭЛ можно практически всегда отнести к одной точке. При выборе точек однородного спектра (15) это уже молчаливо предполагалось, поскольку не учитывались антистоксовые частоты. (Второе оправдание такого выбора - при низких температурах антистоксовое ФК пренебрежимо мало.)

В итоге, предложена и обоснована методика измерения однородной колебательной структуры и факторов Дебая-Валлера в условиях неөднородного уширения электронно-колебательных спектров и решена задача опеделения ФНР, описывающей неоднородный состав примесных центров. Методика, основывающаяся на явлении выжигания провала, применима при низких температурах. Она представляется перспективной для изучения электронно-колебательных взаимодействий и плотности фононных состояний в неупорядоченных примесных средах, а также для исследования статических взаимодействий примеси с матрицей (образование ФНР). Кроме того, относительно полная спектральная характеристика, достигаемая выделением однородных спектров и ФНР, является важной предпосылкой при изучении различных физических явлений с участием электронно-колебательных возбуждений (перенос энергии и др.) в неоднородных системах. Применимость данной методики была экспериментально проверена на системе протохлорофилл в стекле из эфира и бутанола $\left[{ }^{21}\right]$.

Автор выражает благодарность К. К. Ребане за ценные замечания, Р. Авармаа и Я. Кикасу за полезное обсуждение результатов.

\section{ЛИТЕ РА Т У РА}

1. Ребане К. К. Элементарная теория колебательной структуры спектров примесных центров кристаллов. М., «Наука», 1968. 2. Персонов Р. Н., Альшиц Е. И., Быковская Л. А. Письма в ЖЭТФ, 15, № 10, 609-
612 (1972). 3. Гороховский А. А., Каарли Р. К., Ребане Л. А. Письма в ЖЭТФ, 20, № 7, 474-479
(1974).

4. Kharlamov, B. M., Personov, R. I., Bykovskaya, L. A. Opt. Commun., 12, № 2 , $191-193$ (1974).

5. Ребане К. К. Ж. прикл. спектроскопии, 37, № 6, 906-922 (1982)

6. Авармаа Р. Изв. АН ЭССР. Фнз. Матем., 23, № 3, 238-247 (1974).

7. Kikas, J. Chem. Phys. Lett., 57, № 4, 511-513 (1978).

8. Friedrich, J., Haarer, D. J. Chem. Phys., 76, № 1, 61-68 (1982).

9. Кикас Я. Изв. АН ЭССР. Фнз. Матем. 25, № 4, 374-379 (1976). 10. Тамм Т. Б., Кикас Я. В., Сирк А. Э. Ж. прнкл. спектроскопии, 24, № 2, 315-321
(1976).

11. Fünfschilling, J., Zschokke-Gränacher, I., Williams, D. F. J. Chem. Phys., 75, № 8,
3669-3673 (1981).

12. Abram, I. I., Auerbach, R. A., Birge, R. R., Kohler, B. E., Stevenson, J. M. J. Chem. Phys., 63, № 6, 2473-2478 (1975).

13. McColgin, W. C., Marchetti, A. P., Eberly, J. H. J. Amer. Chem. Soc., 100, № 18,
5622-5626 (1978).

14. Gorokhovski, A., Kikas, J. Opt. Commun., 21, № 2, 272-274 (1977)

15. Friedrich, J., Swalen, J. D., Haarer, D. J. Chem. Phys., 73, № 2, 705-711 (1980).

16. Гороховский А. А., Кикас Я. В. Ж. прикл. спектроскопин, 28, № $5,832-838$
(1978).

17. Hayes, J. M., Small, G. J. Chem. Phys., 27, № 1, 151-157 (1978). 
18. Авармаа Р. А., Мауринг К. Х. Опт. и спектр., 41, вып. 4, 670-671 (1976).

19. Kikas, J. V., Treshchalov, A. B. Chem. Phys. Lett., 98, № 4, 295-298 (1983).

20. Bogner, U., Schwarz, R. Phys. Rev. B, 24, № 5, 2846-2849 (1981).

21. Яанисо Р. В., Авармаа Р. А. Ж. прикл. спектроскопии (в печати).

\author{
Институт физики \\ Академии наук Эстонской ССР \\ Поступила в редакцию \\ $27 /$ IV 1984
}

\title{
R. JAANISO \\ HOMOGEENSETE SPEKTRITE JA MITTEHOMOGEENSE JAOTUSFUNKTSIOONI MÄARAMINE AUGUPOLETAMISE MEETODIL
}

On esitatud fotokeemilisel augupōletamisel rajanev metoodika madalatemperatuurilises tahkes maatriksis asuvate lisanditsentrite homogeensete spektraaltunnuste määramiseks ning antud arvutuseeskiri tsentrite jaotuse leidmiseks $0-0$ ülemineku sageduse järgi mõôdetud spektritest. Metoodika on eriti sobiv korrastamata, suure spektraalse mittehomogeensusega lisandisüsteemide uurimiseks.

\section{R. JAANISO}

\section{DETERMINATION OF HOMOGENEOUS SPECTRA AND THE INHOMOGENEOUS DISTRIBUTION FUNCTION BY HOLE BURNING METHOD}

A method based on photochemical hole burning is presented for measuring the singlesite spectra of impurity centres in low-temperature solid matrix. A deconvolution procedure for determining the site-energy distribution from measured spectra is described. The method seems particularly appealing for the investigation of disordered impurity systems with broad inhomogeneous spectra. 